\title{
Fusion in deciduous mandibular anterior teeth- A report of two rare cases
}

\author{
Amina Sultan ${ }^{1, *}$, Akanksha Juneja ${ }^{2}$, Melvin Augustine ${ }^{3}$ \\ ${ }^{\mathbf{1}}$ Associate Professor, ${ }^{2}$ Assistant Professor, Dept. of Dentistry, Jamia Millia Islamia University, New Delhi, ${ }^{3}$ Senior Lecturer, \\ Dept. of Peadiatric \& Preventive Dentistry, Malabar Dental College \& Research Centre, Malappuram, Kerala, India
}

\section{*Corresponding Author: Amina Sultan}

Email: asultan@jmi.ac.in

\begin{abstract}
Dental anomalies such as fusion, germination, double teeth are frequently encountered during routine dental examination often leading to crowding or abnormal spacing in dental arches or increased susceptibility to dental caries and aesthetic problems in pediatric dental patients. Tooth fusion is illustrated when there is the union between dentin and / or enamel of two or more separate developing teeth. Since the fused tooth may pose a wide spectrum of dental problems hence managing such cases requires thorough knowledge and technical skills of dental practitioners. Most of the clinicians advocate minimal intervention technique and preventive approach along with a regular and a long term follow up for the management of fused teeth
\end{abstract}

Keywords: Fusion, Gemination, Double tooth, Dental anomaly, Mandibular incisors, Dentition.

\section{Introduction}

Dental anomalies such as fusion, germination, double teeth are frequently encountered during routine dental examination and often lead to crowding or abnormal spacing in dental arches or increased susceptibility to dental caries along with aesthetic problems in pediatric dental patients. ${ }^{1-3}$ Fusion or double tooth is one the most unusual and a rare developmental anomaly of the shape of the tooth with a hereditary pattern ${ }^{1-4}$ which involves the joining of two developing and separate tooth germs resulting in a single and a wider tooth. ${ }^{2,5-7}$ Fusion may be complete or incomplete depending upon the stage of development and may involve the entire length of teeth or it may involve the roots only, however the dentin is confluent in true fusion. ${ }^{2,5,6}$ Clinically the crown of a fused tooth may be delineated with a notch or groove running bucco-lingually on the entire $\operatorname{crown}^{3,6,7}$ and is often confused with germination especially if fusion involves a supernumerary tooth. ${ }^{3,5,7}$ However a differential diagnosis can be made on the basis of radiographic findings. In a case of fusion, the crowns are united by enamel and /or dentin, having two roots or two root canals systems in a single root, whereas in gemination the two crowns are separated partially or completely with a single root structure. ${ }^{1,9,10}$ Milazzo and Alexander ${ }^{11}$ for the purpose of diagnosis suggested counting of teeth in a dentition. If the number is normal or reduced then it is a case of fusion. ${ }^{11}$

Although this anatomic irregularity can occur in both the deciduous and permanent dentition however the available data in literature indicates that fusion is more common in primary teeth with higher frequency in anterior region. ${ }^{2,4,7-9,12}$ Based on several reports the prevalence of fusion is found to be $0.5-2.5 \%$ in primary dentition and $0.3-0.5 \%$ in permanent dentition. ${ }^{1,8,9}$ The etioogy is unclear and no specific reasons are known. Most of the authors believe that some environmental factors, trauma or lack of space in arches could be responsible for this developmental anomaly. ${ }^{6,13,14}$ No specific or fixed treatment is recommended and different treatment modalities are followed depending upon the requirements of the clinical condition due the fused tooth. ${ }^{3,10}$

According to the published data only few cases of fusion in primary mandibular anterior teeth have been reported in Indian population. ${ }^{6}$ This aim of this article is to report a series of two cases of unilateral fusion of mandibular primary central and lateral incisors with a discussion of their clinical implications and dental management.

\section{Case Reports \\ Case 1}

A 6-year-old boy reported to the department of Pediatric Dentistry, at Faculty of Dentistry, MI with a complaint of crowding in lower arch and eruption of a new tooth lingually. On intra oral examination, the patient was found to be in an early mixed dentition phase with all the permanent molarserupted and left mandibular permanent incisor erupting lingually. During examination it was observed that left primary central and lateral incisor were fused to form a tooth that was larger in size with a deep groove running labio-lingually. The primary mandibular lateral incisor was also missing on left side. (Fig. 1 \& 2) The affected tooth was mobile on palpation and was not affected by any caries and nosign of plaque accumulation was present. Overall hygiene of the patient was satisfactory. The accompanying parent (mother) had a normal dentition and had no family history of any dental anomaly. On radiographic examination it was confirmed that mandibular primary central and lateral incisors were fused on left side. The peri-apical radiograph revealed that crowns and roots of 71 and 72 were fused with complete union of pulp chambers and root canals. Radiographically the fused tooth had large pulp chamber and partial root resorption. (Fig. 3) The 
patient was diagnosed with complete fusion of 71 and 72 with lingual eruption of 31 . The mother and child were unaware of this oral pathology and their main concern was lingual eruption of 31 and crowding in lower arch. Since the patient was above 6 years of age which is natural age of exfoliation of mandibular primary incisors hence extraction of 71 and 72 was done with the consent of parent and put on periodic recalls. The extracted fused tooth had completely joined crowns with a single root that was partially resorbed on the lingual side. (Fig. 4)

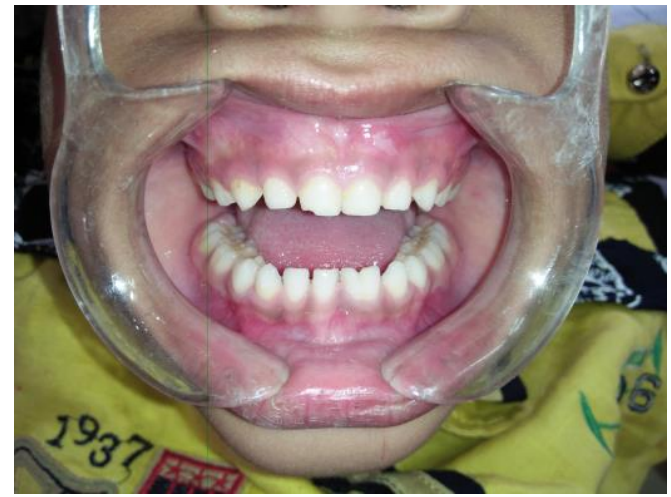

Fig. 1: Fusion between primary mandibular left central and lateral incisors (Intra-oral view)

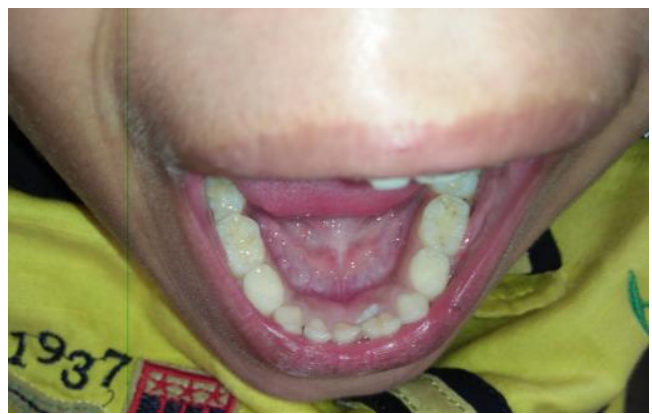

Fig. 2: Intra oral view showing the lingual eruption of 31

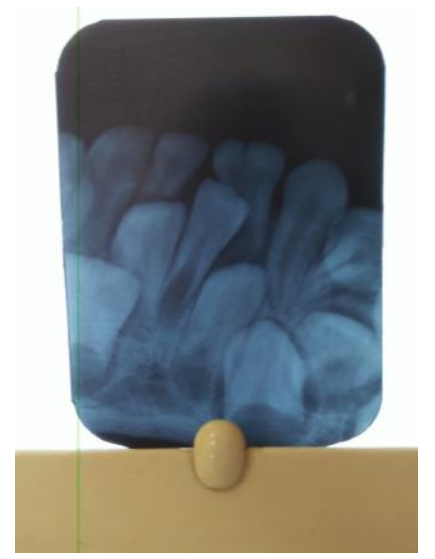

Fig. 3: Intra oral radiograph showing fused primary mandibular left central and lateral incisors with a single root and root canal

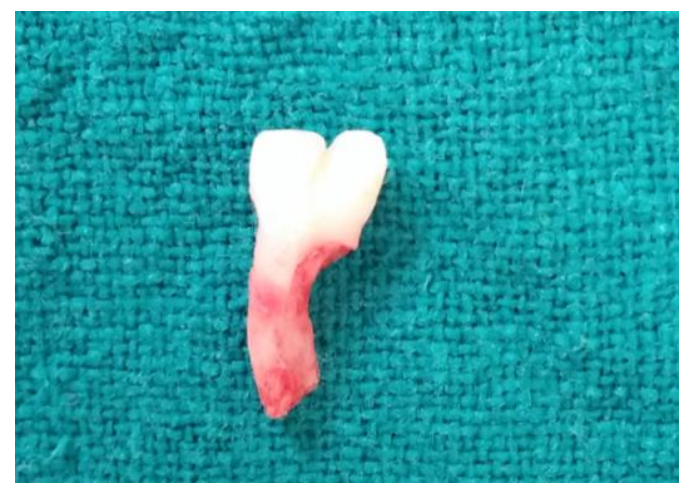

Fig. 4: Extracted fused tooth (71\&72) exhibiting a single large root

\section{Case 2}

A 5-year-old boy reported with a chief complaint of pain and sensitivity in carious teeth for last few days. Intra oral examination revealed that patient had primary dentition. Maxillary primary incisors had dental caries and root stumps were present in relation to 54 . On careful examination of mandibular dentition, it was noted that 81 and 82 were fused together with a characteristic notch present on the incisal edge. (Fig. 5) The fused tooth was firm and free of any signs of smooth surface caries. IOPA radiograph confirmed the diagnosis of fusion of 81 and 82 and the fusion seems to be complete with one root and pulp canal (Fig. 6). During the examination it was also noted that the father of the patient had missing permanent mandibular lateral incisors bilaterallyhence an OPG of the child was taken to detect any hypodontia in succedaneous dentition. The OPG radiograph of the child disclosed a complete set of the permanent dentition with rotation of permanent mandibular right lateral incisor in bone and confirmed the findings of IOPA radiograph. (Fig. 7) The treatment involved the restorations of carious teeth followed by a long term follow up. No treatment was done in the fused tooth as it was asymptomatic. Parents were informed about this irregularity and complications associated with this fused tooth and were given instructions to follow universal preventive regime.

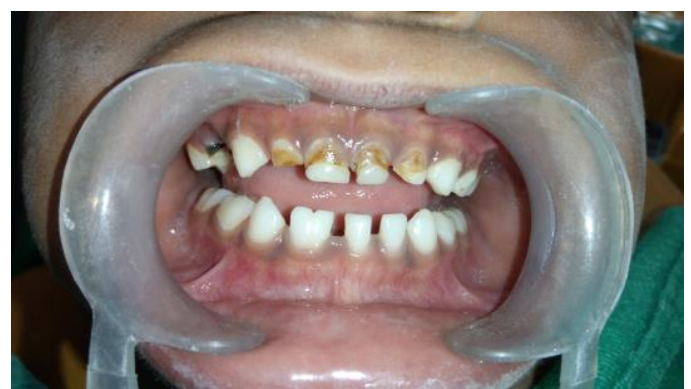

Fig. 5: Fusion between primary mandibular right central and lateral incisors 


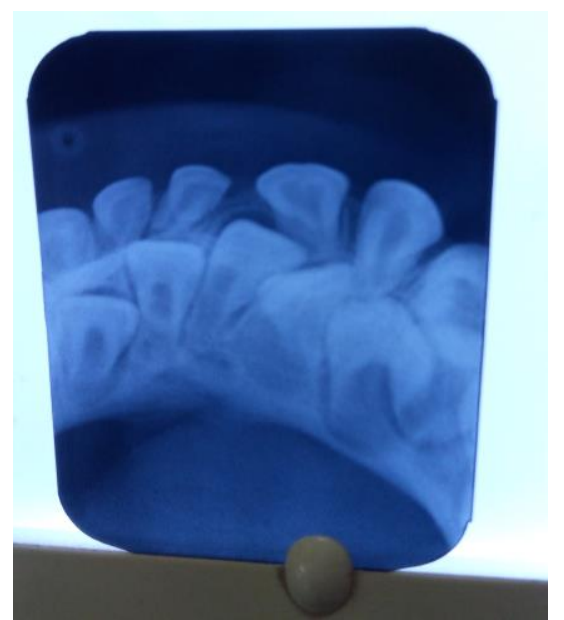

Fig. 6: Intra oral radiograph showing fused primary mandibular right central and lateral incisors with a single root

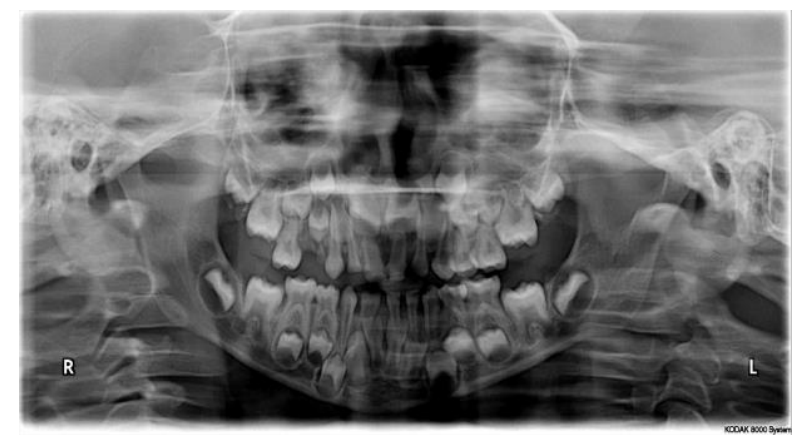

Fig. 7: The OPG radiograph showing fused 81 and 82 and complete set of permanent dentition with rotation in relation to unerupted crown of 42

\section{Discussion}

Tooth fusion is illustrated when there is the union between dentin and /or enamel of two or more separate developing teeth ${ }^{2}$ that can be partial or of total depending uponthe stage of tooth development., ${ }^{2,7,12,14}$ The prevalence of tooth fusion is estimated as $0.5 \%$ $2.5 \%$ in primary dentition with lower prevalence in permanent dentition, $, 1,2,8,9,14$ whereas the prevalence of unilateral double primary teeth is $0.5 \% .{ }^{14}$ Due to this low prevalence the importance of these anomalies tend to be underestimated. ${ }^{1}$ There are several schools of beliefs regarding the etiology of fusion. Most of the researchers suggest that some physical forces or pressure leads to fusion of two tooth germs while some feels this anomaly results due to two tooth buds coming closer and resorbing the interdental bone..$^{2,6,12,14}$ Genetic inheritance can also be considered as one of the cause $\mathrm{s}^{4}$ $6,10,12,14,15$ and in case 2 the father of the patient too had congenitally missing permanent mandibular lateral incisors bilaterally which strengthen this fact.

Fusion of primary teeth is usually asymptomatic but the ramifications of such teeth may result in different types of dental problems. ${ }^{14}$ One of the most common dental implication is aesthetics because of its irregular shape and location in the anterior region. ${ }^{1,3,6,7}$ In both the cases presented, the fused teeth were not located in aesthetic zone and the parents and both the patients were unaware of this phenomenon.

Another dental concern related to fusion of primary teeth is malocclusion including abnormal spacing or crowding in dental arches. The fusion of two teeth requires less space than two normal teeth and this leads to diastema between the fused teeth and adjacent tooth, ${ }^{1,6}$ and this was quite evident in both of our cases. Another clinical implication found frequently in the fused incisors is a groove or notch passing labiolingually vertically through crown surface which can make the tooth susceptible to higher caries risk and plaque accumulation. ${ }^{1,3,6,8,14,16}$ In both the reported cases the fused teeth showed the presence of groove running buccolingually but were caries free and no periodontal problem was diagnosed. Although the second case had all the maxillary incisors and molars carious but the fused tooth was un affected hence the universal preventive regime was followed in this case and was put on regular follow up after the completion of dental treatment. The last and one of the major dental problem encountered in permanent dentition following a fused tooth is the ectopic and delayed eruption of succedaneous anterior teeth. The physiological root resorption of the fused primary incisors may be retarded due to the larger root mass and greater root surface area as compared to the size of the crown of the permanent successor. ${ }^{1,6,8,14}$ This was evident in our first case, where the permanent mandibular left incisor i.e 31 had erupted lingually due to the delayed natural exfoliation of fused 71 and 72 . Since the patient had already attained the age of 6 years which is the natural age of replacement of mandibular primary incisors hence the extraction of the fused tooth was done with the patient consent. Another most common problemreported in $50 \%$ of cases with fused teeth is hypodontia or the repetition of fusion in permanent dentition. ${ }^{1,6,10,17}$ and this was not found in both the cases presented.

Since the fused tooth may pose a wide spectrum of dental problems henceearly diagnosis of this dental anomaly is of significant importance. The dental management of such cases requires thorough knowledge and technical skills of dental practitioners. Most of the clinicians advocate minimal intervention technique and universalpreventive approach along with a regular and a long term follow up for the management of fused teeth. $3,6,14,16$

\section{Conclusion}

Tooth fusion or double tooth is one of the rare and uncommon developmental anomalies of the shape of the tooth. Therefore a early diagnosis and a thorough clinical observation along with radiographs are necessary. A long-term follow-up is very important in most of the cases along with universal preventive procedures and minimal intervention if indicated. 


\section{References}

1. Prasad Rao VA, Reddy NV, Krishnakumar, Sugumaran DK, Mohan G, Senthil, Eagappan AR. Primary double tooth with partial anodontia of permanent dentition - a case report. J Clin Exp Dent. 2010;2(2):e79-81

2. W. G. Shafer, M. K. Hine, and B. M. Levy, A Textbook of Oral Pathology, Elsevier, 5th edition, Pg-54

3. Tewari N, Pandey RK., Bilateral fusion in primary mandibular teeth: a report of two cases. J Indian Soc Pedod Prev Dent. 2011;29(1):50-2.

4. Lewis R. Eversole, Clinical Outline of Oral Pathology: PMPH, USA,4th Edi, pg 572

5. Joseph A. Regezi, James J. Sciubba, Richard C. K. Jordan; Oral Pathology: Clinical Pathologic Correlations in "Abnormalities of teeth" Saunders Publications, 5th edi, pg362.

6. Ansari AA, Pandey P, Gupta VK and Pandey RK. Bilateral Fusion of the Mandibular Primary Incisors with Hypodontia: A Case Report. Austin J Clin Case Rep. 2014;1(12):1057.

7. Seniz Karacay, Gunsel Guven, Ramazan Koymen, Management of a Fused Central Incisor in Association with a Macrodent Lateral Incisor: A Case Report: Pediatric Dentistry. 28:4,2006;336-340.

8. Eliecer Eidelman. Fusion of maxillary primary central and lateral incisors bilaterally. Pediatric Dentistry. 3(4):346-347.

9. B. W. Neville, D. D. Damm, C. M. Allen, and J. E. Bouquet, "Abnormalities of teeth," in Oral and Maxillofacial Pathology, pp. 74-75, WB Saunders, Philadelphia, Pa, USA, 2nd edition, 2002.
10. Yussuf K. Chunawalla, Shweta S. Zingade, Bijle Mohammed Nadeem Ahmed, Pulp therapy in Maxillary fused primary central and lateral incisor: A Case Report Int. Journal of Contemporary Dentistry. 2011;2(2):21-24.

11. Milazzo A, Alexander SA. Fusion, Gemination, Oligodontia and Taurodontism Pedod. 1982;6(2):194199.

12. Amina Sultan, Akanksha Juneja, Sudhanshu Bhatnagar. Bilateral fusion of permanent mandibular central and lateral incisors: A case-report of a rare developmental anomaly. International Journal of Oral Health Dentistry. 2017;3(3):192-194.

13. R. E. McDonald and D. R. Avery, Fusion of teeth, in Dentistry for Child and Adolescent, The C. V. Mosby, St. Louis, Mo, USA, 5th edition, 1983;121-122.

14. BS Rajashekhara et al, Bilateral fusion of primary mandibular lateral incisors and canines: A report of a rare case, Rev. Odontociênc. 2010;25(4):427-429.

15. Harshita Rai, Vaishali Keluskar, AnjanaBagewadi, Bilateral Fusion of Permanent Mandibular Incisors: A Rare Developmental Anomaly IJSS Case Reports \& Reviews. 2016;3(4).

16. Rao PK, Mascarenhas R, Anita A, Devadiga D (2014) Fusion in Deciduous Mandibular Anterior Teeth - A Rare Case. Dentistry S2:001, 2014.

17. R. Veerakumar et al., Caution! We Are Erupting as Twins. Journal of Clinical and Diagnostic Research. 2011;5(5):1123-1124. 